\title{
New formulation of predictors for finite-dimensional linear control systems with input delay
}

\author{
Delphine Bresch-Pietri ${ }^{\mathrm{a}}$, Christophe Prieur $^{\mathrm{b}}$, Emmanuel Trélat ${ }^{\mathrm{c}}$ \\ ${ }^{a}$ MINES Paristech, PSL Research University, CAS - Centre Automatique et Systèmes, 60 \\ bd St Michel 75006 Paris, FRANCE, e-mail: delphine.bresch-pietri@mines-paristech.fr \\ ${ }^{b}$ Université Grenoble Alpes, CNRS, GIPSA-lab, F-38000 Grenoble, France. \\ ${ }^{c}$ Sorbonne Universités, UPMC Univ Paris 06, CNRS UMR 7598, Laboratoire Jacques-Louis \\ Lions, F-75005, Paris, France
}

\begin{abstract}
This paper focuses on a prediction-based control for linear time invariant systems subject to a constant input delay, also known as the Artstein reduction approach. Standardly, this method consists in considering a predicted delay-free system, on which one can design straightforwardly a stabilizing controller. The resulting controller is then defined through an implicit integral equation, involving both the original system state and past values of the input. We propose here an alternative formulation which allows to write explicitly the Artstein transformation, and thus the corresponding controller, in terms of past values of the state only.
\end{abstract}

Keywords: Time-delay systems, Finite Spectrum Assignment, Prediction-based controllers

\section{Introduction}

Even if voluntary delay introduction can sometimes benefit to the control action [20], most of the time, the appearance of delay in control loops is a source of substantial performance degradation, and even of instability if the controller has been designed neglecting this delay (see $[5,6,19]$ for introductions to timedelay systems). Interestingly, these undesirable effects can be circumvented using a predictor-based approach $[1,11,12]$ which enables to recover closedloop performance similar to the delay-free case. The basic idea of this technique grounds on a the use of system state prediction instead of the current state in the control loop, thus compensating for the input delay.

This method has been first introduced for linear time-invariant dynamics subject to a constant input-delay. This is also the framework considered in this paper. It is worth mentioning that numerous improvements and extension of this technique have been proposed in the last decades, such as for nonlinear plants $[7,8,10]$, for various classes of non-constant delays $[2,16]$, for uncertain $[4]$

\footnotetext{
This research was partially supported by the ANR projects MACS-Drill ANR-15-CE230008-01 and Finite4sos ANR-15-CE23-0007-01.
} 
or multiple delays $[3,18]$ or the design of alternative predictions to counteract the effect of integral discretization in the prediction (see the works of [15] on the addition of a low-pass filter or the ones of [23] on truncated predictors or again the ones of [13] on alternative recursive differential predictions).

In this paper, we aim at presenting an alternative formulation of the standard prediction-based technique for constant input delay, the so-called Arststein approach. Standardly, this prediction-based control law is obtained by solving an implicit integral equation involving past values of the input, namely, a Volterra equation of the second kind [17]. Here, we propose to inverse this transformation and obtain an expression of both the Artstein transform and the corresponding controller in terms of the state history only. This is the main contribution of the paper, which aims at providing a new technical tool to study, e.g., implementation issues or robustness properties of prediction-based controllers.

The paper is organized as follows. In Section 2, we briefly recall the Artstein approach before stating our main results, namely, the inversion of the Artstein reduction (see Theorems 1 and 2). Then, we illustrate the interest of this result in different technical applications in Section 3. Finally, Section 4 collects the proofs of all results, whereas some concluding remarks are given in Section 5.

\section{Main results}

\subsection{Standard prediction - Artstein approach}

In this section, we briefly recall the standard Artstein approach.

Consider the following input-delay finite-dimensional linear system

$$
\dot{x}(t)=A x(t)+B u(t-D),
$$

where $x(t) \in \mathbb{R}^{n}, u(t) \in \mathbb{R}^{m}, A$ is a real matrix of size $n \times n, B$ is a real matrix of size $n \times m$ and $D$ is a constant input-delay. In order to stabilize the control system (1), introduce the so-called Artstein model reduction (see [1], see also $[11,12,19])$, i.e., define, for $t \in \mathbb{R}$,

$$
z(t)=x(t)+\int_{t-D}^{t} e^{(t-s-D) A} B u(s) d s
$$

which gives, from an easy computation,

$$
\dot{z}(t)=A z(t)+e^{-D A} B u(t),
$$

that is, a delay-free linear system. Therefore, assuming controllability of the pair $\left(A, e^{-D A} B\right)$, this leads to the natural control choice ${ }^{1}$

$$
u(t)=K_{D} z(t)=K_{D}\left(x(t)+\int_{t-D}^{t} e^{(t-s-D) A} B u(s) d s\right), \quad t \geqslant 0
$$

\footnotetext{
${ }^{1}$ It is interesting to note that the approach (2)-(5) is formally equivalent to ones considering a pole placement in terms of the original dynamics matrices $A, B$ as done, e.g., in [9],
} 
in which the gain matrix $K_{D}$ is chosen such that $A+e^{-D A} B K_{D}$ is Hurwitz. Then, by construction, $t \mapsto z(t)$ converges exponentially to the origin, and hence both $t \mapsto u(t)$ and $t \mapsto \int_{t-D}^{t} e^{(t-D-s) A)} B u(s) d s$ converge exponentially to the origin as well. Then the equality (2) implies that $t \mapsto x(t)$ converges exponentially to the origin.

Theoretically, the predictor-based control (5) stabilizes exponentially the delay control system (1), whatever the value of the delay $D$ may be, and without any restriction on the matrices of the system. This should be put in contrast with the use of a standard proportional controller $u(t)=K x(t)$ which achieves closed-loop stabilization if sufficient conditions bearing on the feedback gain and involving both delay and dynamics matrices are satisfied. Yet, the predictionbased controller (5) is now infinite-dimensional as it involves an integral term depending on past values of the input, the implementation of which can generate serious computational issues [21].

\subsection{Inversion of the Artstein transform}

As emphasized previously, the Artstein transformation and, thus, the corresponding prediction-based control law depend on past values of the control input over a time-horizon $[t-D, t]$. In order to provide an alternative theoretical tool, we propose in this section to invert the Artstein transform (2), that is, to obtain an expression of it depending only on $x(\cdot)$ (and potentially the input over a fixed time-horizon). By expressing both the stabilization feedback law and a Lyapunov functional in terms of the state, we aim at potentially improving robustness margin but also provide new tools to study, e.g., the impact of the discretization of the integral in (5) in an implementation context.

In details, by inverting the Artstein transform, we mean to solve the fixed point implicit equality (5) or, equivalently, to invert the definition of the variable $z$, which, through (2) and (5), satisfies

$$
z(t)=x(t)+\int_{\max (t-D, 0)}^{t} e^{(t-s-D) A} B K_{D} z(s) d s+\int_{t-D}^{\max (t-D, 0)} e^{(t-s-D) A} B u_{0}(s) d s
$$

in which $u_{0}$ denotes the control values for time $t<0$, i.e., $u(t)=u_{0}(t)$ for $t \in[-D, 0)$.

introducing

$$
\begin{aligned}
u(t) & =K\left[e^{D A} x(t)+\int_{t}^{t+D} e^{(t+D-s) A} B u(s-D) d s\right] \\
& =K e^{D A} z(t)
\end{aligned}
$$

Indeed, one formally obtains that the two control laws are similar with $K_{D}=K e^{D A}$. Moreover, noting that

$$
A+e^{-D A} B K_{D}=e^{-D A}(A+B K) e^{D A},
$$

it follows that the closed-loop matrices $A+e^{-D A} B K_{D}$ and $A+B K$ (with $K=K_{D} e^{-D A}$ ) have the same eigenvalues and thus the same stability properties. 
With this aim in view, for every function $f$ defined on $\mathbb{R}$ and locally integrable, we define

$$
\begin{aligned}
& \left(T_{D} f\right)(t)=K_{D} \int_{\max (t-D, 0)}^{t} e^{(t-D-s) A} B f(s) d s \\
& \left(T_{0} f\right)(t)=K_{D} \int_{t-D}^{\max (t-D, 0)} e^{(t-D-s) A} B f(s) d s
\end{aligned}
$$

It follows that (6) can be rewritten as $u(t)=K_{D} x(t)+\left(T_{D} u\right)(t)+\left(T_{0} u_{0}\right)(t)$, for every $t \geqslant 0$. An explicit manual iteration leads to the following expression of the feedback $u$ at time $t$,

$$
\begin{aligned}
u(t) & =K_{D} x(t)+K_{D} \int_{\max (t-D, 0)}^{t} e^{(t-D-s) A} B K_{D} x(s) d s \\
& +K_{D} \int_{t-D}^{\max (t-D, 0)} e^{(t-D-s) A} B u_{0}(s) d s \\
& +K_{D} \int_{\max (t-D, 0)}^{t} e^{(t-D-s) A} B K_{D} \int_{\max (s-D, 0)}^{s} e^{(s-D-\tau) A} B K_{D} x(\tau) d \tau d s \\
& +K_{D} \int_{\max (t-D, 0)}^{t} e^{(t-D-s) A} B K_{D} \int_{s-D}^{\max (s-D, 0)} e^{(s-D-\tau) A} B u_{0}(\tau) d \tau d s+\cdots
\end{aligned}
$$

We summarize more formally this relation in the following theorem (proved in Section 4.1).

Theorem 1. There holds

$$
u(t)= \begin{cases}u_{0}(t) & \text { if } t \in[-D, 0), \\ \sum_{j=0}^{+\infty}\left(T_{D}^{j} K_{D} x\right)(t)+\sum_{j=0}^{+\infty}\left(T_{D}^{j} T_{0} u_{0}\right)(t) & \text { if } t \geqslant 0,\end{cases}
$$

and the series is convergent, whatever the value of the delay $D \geqslant 0$ may be.

Note that, according to this result, the control law at time $t$ depends on past values of $x$ over the time interval $(0, t)$ and on the initial control values over the interval $(-D, 0)$. We reformulate this fact explicitly in the following result (proved in Section 4.2).

Theorem 2. For every $t \in \mathbb{R}_{+}$, there holds

$$
x(t)=z(t)-\int_{0}^{t} \Phi_{D}(t, s) x(s) d s-\int_{-D}^{0} \Phi_{0}(t, s) u_{0}(s) d s
$$

where $\Phi_{D}=0$ if $D=0$ and, otherwise, is defined as, for $(t, s) \in \mathbb{R}_{+}^{2}$,

$$
\Phi_{D}(t, s)=f_{\left\lfloor\frac{t-s}{D}\right\rfloor}\left(t-s-\left\lfloor\frac{t-s}{D}\right\rfloor D\right),
$$

in which $\lfloor\cdot\rfloor$ denotes the integer part of a real number and the sequence of functions $f_{i}:[0, D] \rightarrow \mathcal{M}_{n}(\mathbb{R})$ is defined as follows: 
- $f_{0}$ is the solution of the fixed-point equation

$$
f_{0}(r)=\tilde{f}(r)+\left(\tilde{T}_{0} f_{0}\right)(r), r>0
$$

with, for $r>0$,

$$
\begin{aligned}
\tilde{f}(r) & =e^{(r-D) A} B K_{D} \\
\left(\tilde{T}_{0} f_{0}\right)(r) & =\int_{0}^{r} e^{(r-\tau-D) A} B K_{D} f_{0}(\tau) d \tau
\end{aligned}
$$

- for $i \in \mathbb{N}, f_{i+1}$ is the solution of the fixed-point equation

$$
f_{i+1}(r)=\left(\psi f_{i}\right)(r)+\left(\tilde{T}_{D} f_{i+1}\right)(r), r>0
$$

with, for $r>0$,

$$
\begin{aligned}
\left(\psi f_{i}\right)(r) & =\int_{r}^{D} e^{(r-\tau) A} B K_{D} f_{i}(\tau) d \tau \\
\left(\tilde{T}_{D} f_{i+1}\right)(r) & =\int_{0}^{r} e^{(r-\tau-D) A} B K_{D} f_{i+1}(\tau) d \tau
\end{aligned}
$$

and, finally, $\Phi_{0}=0$ if $D=0$ and, otherwise, is defined as, for $(t, s) \in \mathbb{R}_{+}^{2}$,

$$
\Phi_{0}(t, s)=g_{\left\lfloor\frac{t}{D}\right\rfloor}(t, s)
$$

in which the sequence of functions $g_{i}: \mathbb{R}_{+} \times[-D, 0] \rightarrow \mathcal{M}_{n}(\mathbb{R})$ are given as follows

- $g_{0}$ is the solution to the fixed-point equation

$$
g_{0}(t, s)=\left\{\begin{array}{cl}
\tilde{f}(t-s)+\left(\check{T}_{0,1} g_{0}\right)(t, s), & \text { if } s \in[t-D, 0] \\
\left(\check{\psi}_{0} g_{0}\right)(t, s)+\left(\check{T}_{0,2} g_{0}\right)(t, s), & \text { if } s \in[-D, t-D]
\end{array}\right.
$$

with $\tilde{f}$ defined in (12) and

$$
\begin{aligned}
\left(\check{T}_{0,1} g_{0}\right)(t, s) & =\int_{0}^{t} e^{(t-\tau-D) A} B K_{D} g_{0}(\tau, s) d \tau \\
\left(\check{\psi}_{0} g_{0}\right)(t, s) & =\int_{0}^{s+D} e^{(t-\tau-D) A} B K_{D} g_{0}(\tau, s) d \tau \\
\left(\check{T}_{0,2} g_{0}\right)(t, s) & =\int_{s+D}^{t} e^{(t-\tau-D) A} B K_{D} g_{0}(\tau, s) d \tau
\end{aligned}
$$

- for $i \in \mathbb{N}, g_{i+1}$ is the solution to the fixed-point equation

$$
g_{i+1}(t, s)=\left(\check{\psi}_{i} g_{i}\right)(t, s)+\left(\check{T}_{i} g_{i+1}\right)(t, s)
$$


with

$$
\begin{aligned}
\left(\check{\psi}_{i} g_{i}\right)(t, s) & =\int_{t-D}^{i D} e^{(t-\tau-D) A} B K_{D} g_{i}(\tau, s) d \tau \\
\left(\check{T}_{i} g_{i+1}\right)(t, s) & =\int_{i D}^{t} e^{(t-\tau-D) A} B K_{D} g_{i+1}(\tau, s) d \tau
\end{aligned}
$$

Consequently, the prediction-based control law (5) can be formulated as

$$
u(t)=K_{D} x(t)+K_{D} \int_{0}^{t} \Phi_{D}(t, s) x(s) d s+K_{D} \int_{-D}^{0} \Phi_{0}(t, s) u_{0}(s) d s, \quad t \geqslant 0
$$

One can of course notice that the form of this last expression is consistent with the one provided in (8) and which was derived in Theorem 1.

\subsection{Artstein transformation inversion: alternative expression of the kernels}

In view of implementation, it is worth mentioning that (11)-(14) and (16)(17) can equivalently be written under a differential form as follows.

Corollary 1. Consider $D>0$. The functions $f_{i}$ and $g_{i}, i \in \mathbb{N}$, introduced in Theorem 2 satisfy

$$
\begin{aligned}
& \dot{f}_{0}(r)=\left(A+e^{-A D} B K_{D}\right) f_{0}(r), \quad f_{0}(0)=e^{-A D} B K_{D} \\
& \dot{f}_{1}(r)=\left(A+e^{-A D} B K_{D}\right) f_{1}(r)-B K_{D} f_{0}(r), \quad f_{1}(0)=f_{0}(D)-B K_{D} \\
& \dot{f}_{i+1}(r)=\left(A+e^{-A D} B K_{D}\right) f_{i+1}(r)-B K_{D} f_{i}(r), \quad f_{i+1}(0)=f_{i}(D), \quad i \in \mathbb{N}^{*}
\end{aligned}
$$

and

$$
\begin{aligned}
& \partial_{t} g_{0}(t, s)=\left(A+e^{-D A} B K_{D}\right) g_{0}(t, s) \\
& g_{0}(0, s)=e^{-(s+D) A} B, \quad g_{0}\left(t,(t-D)^{-}\right)=B+g_{0}\left(t,(t-D)^{+}\right) \\
& \partial_{t} g_{i}(t, s)=\left(A+e^{-D A} B K_{D}\right) g_{i}(t, s)-B K_{D} g_{i-1}(t-D, s) \\
& g_{i}(i D, s)=g_{i-1}(i D, s), \quad i \in \mathbb{N}^{*}
\end{aligned}
$$

The proof of this Corollary follows from a straightforward differentiation of the fixed point equations listed in Theorem 2.

Remark 1. Interestingly enough, (19)-(21) imply that $\operatorname{rank}\left(\Phi_{D}(t, s)\right) \leqslant 1$ for all $(t, s) \in \mathbb{R}_{+}^{2}$, at least for the scalar control case $(m=1)$. Similarly to the inversion of (6) carried out in Theorem 1 and 2, if one wishes to invert the relation

$$
u(t)=K_{D} x(t)+K_{D} \int_{t-D}^{t} e^{A(t-s-D) A} B u(s) d s
$$


under the form (assuming, without loss of generality, that $u_{0}=0$ )

$$
K_{D} x(t)=u(t)-\int_{0}^{t} \Psi_{D}(t, s) K_{D} x(s) d s
$$

then, it follows that

$$
K_{D} \Phi_{D}(t, s)=\Psi_{D}(t, s) K_{D}
$$

that is, $K_{D}^{T}$ is an eigenvector of $\Phi_{D}(t, s)^{T}$ associated with the eigenvalue $\Psi_{D}(t, s)$, in the scalar case $m=1$. This geometrical property might be of interest for implementation purposes and thus could be worth investigating in future works.

\section{Illustration and numerical simulations}

In this section, we aim at illustrating the merits of the Artstein inversion proposed in Theorems 1-2 (and Corollary 1) by considering some cases of potential technical applications.

\subsection{Numerical example}

We first illustrate numerically the convergence properties obtained with a prediction-based controller expressed with the alternative Artstein inversion and discuss some related implementation issues.

We consider the following system

$$
\dot{x}(t)=A x(t)+B u(t-D)
$$

where $A$ is the unstable matrix defined by $A=0.01\left(\begin{array}{cc}10 & 10 \\ 1 & 1\end{array}\right), B=\left(\begin{array}{l}1 \\ 0\end{array}\right)$ and $D=1$ is a constant delay. The gain matrix $K_{D}=-\left(\begin{array}{ll}1.66 & 31.4\end{array}\right)$ is chosen such that the delay-free closed-loop eigenvalues of $A+e^{-A D} B K_{D}$ are -0.5 and -0.6 respectively. The initial conditions are chosen as $x(0)=\left(\begin{array}{c}1 \\ -1\end{array}\right)$ and $u_{0}(\cdot)=0$.

We chose to implement the control law (18) based on the differential expressions proposed in Corollary 1, using a discrete time step $h=0.01 \mathrm{~s}$. Corresponding time-evolutions of the closed-loop system are given in Figure 1. Asymptotic convergence is well achieved and transient performances are identical to the ones that would have obtained using the original formulation (5), as was expected.

It is worth mentioning the fact that, as the feedback law (18) requires the knowledge of the state over the time horizon $(0, t)$, it is not directly implementable as it requires to store a number of information linearly increasing with time. Furthermore, numerical approximations resulting from discretization result into a finite-time explosion, i.e., stability of the closed-loop discretized scheme only holds up to a reliability time $T^{\star}$ after which the system diverges 
(see Appendix for more details). This reliability time $T^{*}$ can be taken arbitrarily large, but only at the expense of the computational burden by selecting the time step $h$ arbitrarily small.

Note that this interesting feature does not depend on the chosen implementation technique of the Artstein inversion. If one had chosen to rely on the fixed-point equations (11)-(17), an equivalent tradeoff exist between reliability time and the number of iterations used to solve the fixed-point equations. Similarly, if one implements the inversion with the infinite sum expression of Theorem 1, the tradeoff would have concerned the truncation order of the infinite sum.
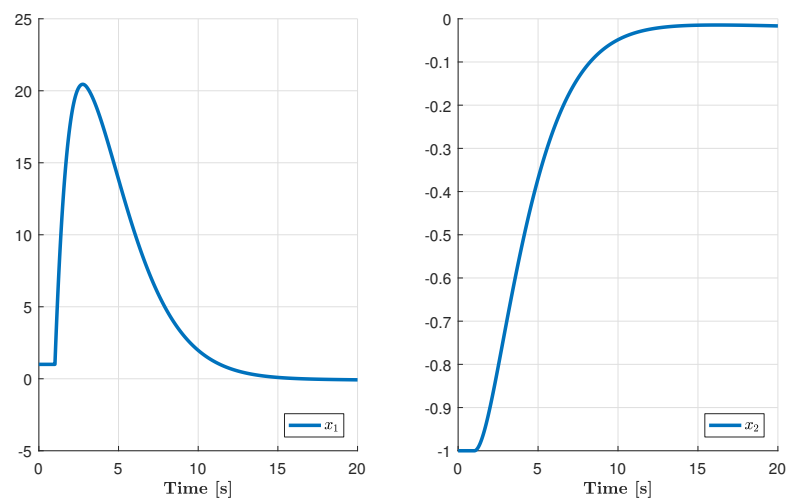

(a) State components $x_{1}$ (left) and $x_{2}$ (right).

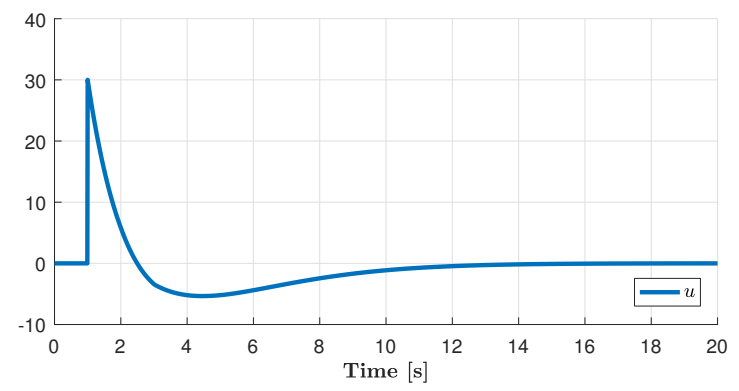

(b) Control $u$ given by (18) in Theorem 2 .

Figure 1: Time-evolution of the closed-loop system consisting of the plant given in Section 3.1 and the control law (18). The gain matrix $K_{D}$ is chosen such that the delay-free closed-loop eigenvalues are -0.5 and -0.6 respectively. The initial conditions are chosen as $x(0)=[1-1]^{T}$ and $u_{0}(\cdot)=0$.

Thus, to counteract this effect, in simulation, we virtually reset the initial time $t_{0}$ periodically (every 2 seconds here), with a period smaller than $T^{\star}$. Consequently, the implemented controller only requires the history of the state over a bounded time horizon (and still of the input over a fixed time horizon) and can be implemented accurately with a time step limiting the computational 
burden.

\subsection{Explicit effect of integral discretization}

Conversely, if we focus on the implementation of the standard expression (5) of a prediction-based controller, a critical issue concerns the effect of discretization of the integral in (5), that is, the use in practice of the discrete form

$$
u(t)=K_{D}\left[x(t)+\sum_{i \in \mathcal{I}_{n}} h_{i} e^{A \theta_{i}} B u\left(t-\theta_{i}\right)\right]
$$

where $\mathcal{I}_{n}$ is a finite sequence of sets of length $\left(h_{i}\right)$ mapping the interval $[-D, 0]$ and the scalars $\theta_{i}$ depend on the selected integration rule.

As was first shown in [21] and thoroughly investigated in [14, 15], the closedloop system consisting of (1) and the discretized control law (26) may be unstable for arbitrarily large values of $n$. This striking fact can be understood using eigenvalue considerations. Indeed, when the theoretical control law (5) is replaced by the approximated form (26), the finite spectrum property is lost and the corresponding state-delayed differential equation possesses an infinite number of characteristic roots, some of them potentially tending to the right-half complex plane. However, determining these characteristic roots is potentially complex.

Theorem 2 reveals helpful in this context as it allows to obtain directly the corresponding Delay Differential Equation and characteristic equations. Namely, assuming $u_{0}(\cdot)=0$ for the sake of simplicity and using (9), one can straightforwardly rewrite (1) under the form

$\dot{x}(t)=A x(t)+B K_{D} x(t-D)+B K \sum_{i \in \tilde{\mathcal{I}}_{n}} h_{i} \Phi_{D}\left(t, t-\theta_{i}\right) x\left(t-\theta_{i}-D\right) d s, \quad t \geqslant D$

where $\tilde{\mathcal{I}}_{n}$ is a finite sequence of sets of length $\left(h_{i}\right)$ mapping the interval $[0, t]$, the characteristic equation of which can be studied numerically. Thus, Theorem 2 provides a constructive procedure to study the closed-loop stability resulting from a given integral discretization scheme.

\subsection{Alternative proof of closed-loop stability}

Finally, we show how the exponential stability of the Arstein's transform can be obtained independently from the arguments previously used in Section 2.1, using only the definitions and properties introduced in Theorem 2.

Corollary 2. The variable $z$ defined through (9) as

$$
z(t)=x(t)+\int_{0}^{t} \Phi_{D}(t, s) x(s) d s+\int_{-D}^{0} \Phi_{0}(t, s) u_{0}(s) d s
$$


satisfies

$$
\dot{z}=\left(A+e^{-A D} B K_{D}\right) z, \quad t \geqslant 0
$$

and thus converges exponentially to the origin.

Proof. First, consider that $t \leqslant D$. Then, due the definitions of $\Phi_{D}$, one simply has

$$
z(t)=x(t)+\int_{0}^{t} f_{0}(t-s) x(s) d s+\int_{-D}^{0} g_{0}(t, s) u_{0}(s) d s
$$

Taking a time-derivative of (15)-(16), one can write

$$
\begin{aligned}
\dot{z}(t)= & A x(t)+B u(t-D)+f_{0}(0) x(t)+\int_{0}^{t} \dot{f}_{0}(t-s) x(s) d s \\
& +\int_{-D}^{0} \partial_{t} g_{0}(t, s) u_{0}(s) d s-B u_{0}(t-D)
\end{aligned}
$$

Using Corollary 1, it follows that

$$
\dot{z}(t)=\left(A+B K_{D}\right) z(t)+B u(t-D)-B u_{0}(t-D), \quad t \in[0, D]
$$

which gives $(28)$ as $u(t)=u_{0}(t)$ for $t \leqslant 0$. Now, consider that $t>D$. We start by observing that, due to the definition of $\Phi_{D}$, one can write

$$
z(t)=x(t)+\sum_{i=0}^{\left\lfloor\frac{t}{D}\right\rfloor} \int_{\max \{0, t-(i+1) D\}}^{t-i D} f_{i}(t-s-i D) x(s) d s+\int_{-D}^{0} g_{\left\lfloor\frac{t}{D}\right\rfloor}(t, s) u_{0}(s) d s
$$

Taking a time-derivative of this last expression, one gets

$$
\begin{aligned}
\dot{z}(t)= & A x(t)+B u(t-D)+f_{0}(0) x(t)+\sum_{i=0}^{\left\lfloor\frac{t}{D}-1\right\rfloor}\left[f_{i+1}(0)-f_{i}(D)\right] x(t-(i+1) D) \\
& +\sum_{i=0}^{\left\lfloor\frac{t}{D}\right\rfloor} \int_{\max \{0, t-(i+1) D\}}^{t-i D} \dot{f}_{i}(t-s-i D) x(s) d s+\int_{-D}^{0} \partial_{t} g_{\left\lfloor\frac{t}{D}\right\rfloor}(t, s) u_{0}(s) d s
\end{aligned}
$$

Hence, with Corollary 1, it follows that

$$
\begin{aligned}
\dot{z}(t)= & \left(A+e^{-A D} B K_{D}\right) z(t)+B u(t-D)-B K_{D} x(t-D) \\
& -B K_{D} \sum_{i=1}^{\left\lfloor\frac{t}{D}\right\rfloor} \int_{\max \{0, t-(i+1) D\}}^{t-i D} f_{i-1}(t-s-i D) x(s) d s \\
& -B K_{D} \int_{-D}^{0} g_{\left\lfloor\frac{t-D}{D}\right\rfloor}(t-D, s) u_{0}(s) d s .
\end{aligned}
$$


As the choice of the control law yields, for $t \geqslant D$,

$$
\begin{aligned}
B u(t-D)=B K_{D} z(t-D) & \\
= & B K_{D} x(t-D)+B K_{D} \sum_{i=0}^{\left\lfloor\frac{t-D}{D}\right\rfloor} \int_{\max \{0, t-(i+2) D\}}^{t-(i+1) D} f_{i}(t-D-s-i D) x(s) d s \\
& +B K_{D} \int_{-D}^{0} g_{\left\lfloor\frac{t-D}{D}\right\rfloor}(t-D, s) u_{0}(s) \\
= & B K_{D} x(t-D)+B K_{D} \sum_{i=1}^{\left\lfloor\frac{t}{D}\right\rfloor} \int_{\max \{0, t-(i+1) D\}}^{t-i D} f_{i-1}(t-s-i D) x(s) \\
& +B K_{D} \int_{-D}^{0} g_{\left\lfloor\frac{t-D}{D}\right\rfloor}(t-D, s) u_{0}(s) d s
\end{aligned}
$$

it follows that

$$
\dot{z}(t)=\left(A+e^{-A D} B K_{D}\right) z(t), \quad t \geqslant 0
$$

Thus, using the fact that $K_{D}$ is chosen such that $A+e^{-A D} B K_{D}$ is Hurwitz, one concludes that $z$ converges exponentially to the origin.

\section{Proofs}

\subsection{Proof of Theorem 1}

We define the functions $\varphi_{D j}$ iteratively by

$$
\begin{aligned}
\varphi_{D_{1}}(t, \tau) & =1 \\
\varphi_{D_{j+1}}(t, \tau) & =\int_{\max (\tau, t-D)}^{\min (t, \tau+j D)} \varphi_{D_{j}}(s, \tau) d s, \quad j \in \mathbb{N}^{*},
\end{aligned}
$$

for every $t \geqslant \tau$, and by $\varphi_{D_{j}}(t, \tau)=0$ if $t<\tau$ and $j \in \mathbb{N}$.

Let us prove by induction that

$$
\left|\left(T_{D}^{j} K_{D} x\right)(t)\right| \leqslant\|B\|^{j}\left\|K_{D}\right\|^{j+1} \int_{\max (t-j D, 0)}^{t} \varphi_{D j}(t, \tau) e^{-(t-j D-\tau)\|A\|}\|x(\tau)\| d \tau,
$$

for every $j \in \mathbb{N}^{*}$. This is clearly true for $j=1$, since

$$
\begin{aligned}
\left|\left(T_{D} K_{D} x\right)(t)\right| & =\left|K_{D} \int_{\max (t-D, 0)}^{t} e^{(t-D-s) A} B K_{D} x(s) d s\right| \\
& \leqslant\|B\|\left\|K_{D}\right\|^{2} \int_{\max (t-D, 0)}^{t} e^{-(t-D-s)\|A\|}\|x(s)\| d s .
\end{aligned}
$$


Assume that this is true for an integer $j \in \mathbb{N}^{*}$, and let us derive the estimate for $j+1$. Since

$$
\left(T_{D}^{j+1} K_{D} x\right)(t)=K_{D} \int_{\max (t-D, 0)}^{t} e^{(t-D-s) A} B\left(T_{D}^{j} K_{D} x\right)(s) d s,
$$

we get

$$
\begin{aligned}
& \left|\left(T_{D}^{j+1} K_{D} x\right)(t)\right| \leqslant\|B\|^{j+1}\left\|K_{D}\right\|^{j+2} \times \\
& \quad \times \int_{\max (t-D, 0)}^{t} e^{-(t-D-s)\|A\|} \int_{\max (s-j D, 0)}^{s} \varphi_{D j}(s, \tau) e^{-(s-j D-\tau)\|A\|}\|x(\tau)\| d \tau d s,
\end{aligned}
$$

and, from the Fubini theorem, noting that $(\tau, s)$ is such that

$$
\max (s-j D, 0) \leqslant \tau \leqslant s, \quad \max (t-D, 0) \leqslant s \leqslant t,
$$

if and only if

$$
\max (t-(j+1) D, 0) \leqslant \tau \leqslant t, \quad \max (\tau, t-D) \leqslant s \leqslant \min (t, \tau+j D),
$$

we get the estimate

$$
\begin{aligned}
& \left|\left(T_{D}^{j+1} K_{D} x\right)(t)\right| \leqslant\|B\|^{j+1}\left\|K_{D}\right\|^{j+2} \times \\
& \quad \int_{\max (t-(j+1) D, 0)}^{t}\left(\int_{\max (\tau, t-D)}^{\min (t, \tau+j D)} \varphi_{D j}(s, \tau) d s\right) e^{-(t-(j+1) D-\tau)\|A\|}\|x(\tau)\| d \tau,
\end{aligned}
$$

and the desired estimate for $j+1$ follows by definition of $\varphi_{D_{j+1}}$.

Now, we claim that

$$
0 \leqslant \varphi_{D_{j}}(t, \tau) \leqslant \frac{(t-\tau)^{j-1}}{(j-1) !}
$$

for every $j \in \mathbb{N}^{*}$. Indeed, the nonnegativity is obvious, and the right-hand side estimate easily follows from the fact that $\varphi_{D_{j+1}}(t, \tau) \leqslant \int_{\tau}^{t} \varphi_{D_{j}}(s, \tau) d s$ and from a simple iteration argument.

Finally, from (30) and (31), we infer that

$$
\begin{aligned}
\left|\left(T_{D}^{j} K_{D} x\right)(t)\right| & \leqslant\|B\|^{j}\left\|K_{D}\right\|^{j+1} \int_{\max (t-j D, 0)}^{t} \frac{(t-\tau)^{j-1}}{(j-1) !} e^{-(t-j D-\tau)\|A\|}\|x(\tau)\| d \tau \\
& \leqslant\|B\|^{j}\left\|K_{D}\right\|^{j+1} \frac{\left(t e^{D\|A\|}\right)^{j}}{j !} \max _{0 \leqslant s \leqslant t}\|x(s)\|,
\end{aligned}
$$

whence the convergence of the series in (8).

Using similar arguments, one can obtain by induction that

$$
\left|\left(T_{D}^{j} T_{0} u_{0}\right)(t)\right| \leqslant\|B\|^{j+1}\left\|K_{D}\right\|^{j+1} D \frac{\left(t e^{D\|A\|}\right)^{j}}{j !} \max _{s \in[-D, 0]}\left|u_{0}(s)\right|
$$

and the convergence of the second series in (8) follows. 


\subsection{Proof of Theorem 2}

When $D=0$, as $x=z$ in view of Theorem 1 , Theorem 2 straightforwardly holds.

When $D>0$, let us search the kernels $\Phi_{D}$ and $\Phi_{0}$ such that there holds

$$
x(t)=z(t)-\int_{0}^{t} \Phi_{D}(t, s) x(s) d s-\int_{-D}^{0} \Phi_{0}(t, s) u_{0}(s) d s,
$$

postulating, in view of (7), that $\Phi_{D}(t, s)=0$ whenever $s<0$ or $s>t$ and that $\Phi_{0}(t, s)=0$ for $s>0$ or $s<-D$. When defining $\Phi_{D}$ and $\Phi_{0}$ in the sequel, we do not consider sets of null Lebesgue measure, since it does not impact the integral in (9). Namely, in the following, we omit to define $\Phi_{D}$ and $\Phi_{0}$ for $t-s=n D$, $n \in \mathbb{N}$. Using (2) and (5), we must have, for all $t \in \mathbb{R}_{+}$,

$$
\int_{0}^{t} \Phi_{D}(t, s) x(s) d s=\int_{(t-D, t) \cap(0,+\infty)} e^{(t-s-D) A} B K_{D}\left(x(s)+\int_{0}^{s} \Phi_{D}(s, \tau) x(\tau) d \tau\right) d s
$$

and

$$
\begin{aligned}
\int_{-D}^{0} \Phi_{0}(t, s) u_{0}(s) d s= & \int_{\max (t-D, 0)}^{t} e^{(t-s-D) A} B K_{D} \int_{-D}^{0} \Phi_{0}(s, \tau) u_{0}(\tau) d \tau d s \\
& +\int_{t-D}^{\max (t-D, 0)} e^{(t-s-D) A} B u_{0}(s) d s
\end{aligned}
$$

In a first move, we focus on (32) which, using Fubini theorem, rewrites

$$
\begin{aligned}
\int_{0}^{t} \Phi_{D}(t, s) x(s) d s= & \int_{\max (t-D, 0)}^{t} e^{(t-s-D) A} B K_{D} x(s) d s \\
& +\int_{0}^{t} \int_{\max (t-D, s)}^{t} e^{(t-\tau-D) A} B K_{D} \Phi_{D}(\tau, s) d \tau x(s) d s .
\end{aligned}
$$

Since this equality should hold for every $x$,

$$
\begin{aligned}
\Phi_{D}(t, s)= & e^{(t-s-D) A} B K_{D} \chi_{(\max (t-D, 0), t)}(s) \\
& +\int_{\max (t-D, s)}^{t} e^{(t-\tau-D) A} B K_{D} \Phi_{D}(\tau, s) d \tau
\end{aligned}
$$

Let us now solve the implicit equation (33), following two cases depending on the value of $t$.

1. First of all, if $0<t<D$ then $\max (t-D, 0)=0$ and (33) yields

$$
\Phi_{D}(t, s)=e^{(t-s-D) A} B K_{D} \chi_{(0, t)}(s)+\int_{s}^{t} e^{(t-\tau-D) A} B K_{D} \Phi_{D}(\tau, s) d \tau .
$$

There are two subcases for the value of $s$. 
(a) If $s<0$ or if $s>t$ then clearly $\Phi_{D}(t, s)=0$ is a solution.

(b) If $0<s<t$ then

$$
\Phi_{D}(t, s)=e^{(t-s-D) A} B K_{D}+\int_{0}^{t-s} e^{(t-s-\tau-D) A} B K_{D} \Phi_{D}(\tau+s, s) d \tau
$$

and then setting $r=t-s$ (note that $0<r<t<D$ ) we search $\Phi_{D}(t, s)=f_{0}(r)$ with

$$
f_{0}(r)=e^{(r-D) A} B K_{D}+\int_{0}^{r} e^{(r-\tau-D) A} B K_{D} f_{0}(\tau) d \tau,
$$

that is, $f_{0}(r)=\tilde{f}(r)+\left(\tilde{T}_{0} f_{0}\right)(r)$ as stated in (11)-(13).

2. If $t>D$ then $\max (t-D, 0)=t-D$ and (33) yields

$$
\Phi_{D}(t, s)=e^{(t-s-D) A} B K_{D} \chi_{(t-D, t)}(s)+\int_{\max (s, t-D)}^{t} e^{(t-\tau-D) A} B K_{D} \Phi_{D}(\tau, s) d \tau
$$

and we have $\left\lfloor\frac{t}{D}\right\rfloor+2$ subcases for the value of $s$.

(a) If $s<0$ or if $s>t$ then clearly $\Phi_{D}(t, s)=0$ is a solution.

(b) If $t-D<s<t$ then, following the exact same arguments as previously, one can show that $\Phi_{D}(t, s)=f_{0}(t-s)$ in which $f_{0}$ has been previously introduced as the solution of fixed-point equation $f_{0}=\tilde{f}+\tilde{T}_{0} f_{0}$ given in (11)-(13).

(c) If $t-2 D<s<t-D$, then

$$
\Phi_{D}(t, s)=\int_{t-D}^{t} e^{(t-\tau-D) A} B K_{D} \Phi_{D}(\tau, s) d \tau
$$

and, from the previous subcase, one obtains

$$
\begin{aligned}
\Phi_{D}(t, s)= & \int_{t-D}^{s+D} e^{(t-\tau-D) A} B K_{D} f_{0}(\tau-s) d \tau \\
& +\int_{s+D}^{t} e^{(t-\tau-D) A} B K_{D} \Phi_{D}(\tau, s) d \tau
\end{aligned}
$$

Define $\Phi_{D}(t, s)=f_{1}(t-s-D)$ and $r=t-s-D \in[0, D]$. Then, (35) rewrites

$$
f_{1}(r)=\int_{r}^{D} e^{(r-\xi) A} B K_{D} f_{0}(\xi) d \xi+\int_{0}^{r} e^{(r-\xi-D) A} B K_{D} f_{1}(\xi) d \xi
$$

and, thus, $f_{1}$ is the solution of the following fixed-point equations

$$
f_{1}(r)=\left(\psi f_{0}\right)(r)+\left(\tilde{T}_{D} f_{1}\right)(r), r>0
$$

with, for $r>0$,

$$
\begin{gathered}
\left(\psi f_{0}\right)(r)=\int_{r}^{D} e^{(r-\xi) A} B K_{D} f_{0}(\xi) d \xi \\
\left(\tilde{T}_{D} f_{1}\right)(r)=\int_{0}^{r} e^{(r-\xi-D) A} B K_{D} f_{1}(\xi) d \xi
\end{gathered}
$$

in which $f_{0}$ has been previously introduced. 
(d) There remains $\left\lfloor\frac{t}{D}\right\rfloor-1$ subcases for the value of $s$. Those can be straightforwardly investigated with the same arguments and an iteration procedure, as, in particular, the implicit equation (14) which is obtained does not depend on the index $i \in \mathbb{N}$. This concludes the definition of $\Phi_{D}$.

We now have to investigate (33) which, using Fubini Theorem, rewrites

$$
\begin{aligned}
\int_{-D}^{0} \Phi_{0}(t, s) u_{0}(s) d s= & \int_{-D}^{0} \int_{(t-D, t) \cap(0,+\infty)} e^{(t-\tau-D) A} B K_{D} \Phi_{0}(\tau, s) u_{0}(s) d \tau d s \\
& +\int_{t-D}^{\max (t-D, 0)} e^{(t-s-D) A} B u_{0}(s) d s
\end{aligned}
$$

As this equality should hold for every $u_{0}$, one gets

$$
\Phi_{0}(t, s)=\int_{\max (t-D, 0)}^{t} \stackrel{(t-\tau-D) A}{B} K_{D} \Phi_{0}(\tau, s) d \tau+e^{(t-s-D) A} B \chi_{(t-D, \max (t-D, 0))}(s) .
$$

Consider first that $t \in[0, D]$ and that $s \in[t-D, 0]$, then this last equality rewrites

$$
\Phi_{0}(t, s)=\int_{0}^{t} e^{(t-\tau-D) A} B K_{D} \Phi_{0}(\tau, s) d \tau+e^{(t-s-D) A} B
$$

in which $s \geqslant \tau-D$ for $\tau \in[0, t]$ as $s \geqslant t-D$. This gives (16) which solution is unique and converges using the same arguments as previously.

Similar straightforward considerations on the kernel $\Phi_{0}$ conclude the proof of Theorem 2.

\subsection{Proof of Remark 1}

In this subsection, we assume throughout that $m=1$ and, for the sake of simplicity of the exposition, that $u_{0}=0$.

We first show that $\operatorname{rank}\left(f_{i}(r)\right) \leqslant 1, i \in \mathbb{N}$.

Indeed, $B$ is a column and $K_{D}$ is a row, then $B K_{D}$ has rank one. Multiplying by an invertible matrix does not change the rank, thus $f_{0}(0)=e^{-D A} B K_{D}$ has rank one, and then $f_{0}(r)=e^{r\left(A+e^{-D A} B K_{D}\right)} f_{0}(0)$, according to Corollary 1 , has rank one.

Now, from (19)-(20), one notices that

$$
f_{1}(0)=\int_{0}^{D} e^{-s A} B K_{D} f_{0}(s) d s=\int_{0}^{D} e^{-s A} B K_{D} e^{s \tilde{A}} d s f_{0}(0)=C f_{0}(0),
$$


where we have set $\tilde{A}=A+e^{-D A} B K_{D}$ and $C=\int_{0}^{D} e^{-s A} B K_{D} e^{s \tilde{A}} d s$. Hence ${ }^{2}$, $\operatorname{rank}\left(f_{1}(0)\right) \leqslant 1$. Besides, integrating $(20)$, we have

$$
\begin{aligned}
f_{1}(r) & =e^{r \tilde{A}} f_{1}(0)-\int_{0}^{r} e^{(r-s) \tilde{A}} B K_{D} f_{0}(s) d s \\
& =\left(e^{r \tilde{A}} C-\int_{0}^{r} e^{(r-s) \tilde{A}} B K_{D} e^{s \tilde{A}} d s\right) f_{0}(0)
\end{aligned}
$$

and thus $\operatorname{rank}\left(f_{1}(r)\right) \leqslant 1$. The desired conclusion follows by direct iteration using (21).

Now, if we consider

$$
K_{D} x(t)=u(t)-\int_{0}^{t} \psi_{D}(t, s) K_{D} x(s) d s,
$$

then, a reasoning similar to the one made in Section 4.2 leads to

$$
\psi_{D}(t, s)=K_{D} e^{(t-s-D) A} B \chi_{(\max (t-D, 0), t)}(s)+K_{D} \int_{\max (s, t-D, 0)} e^{(t-\tau-D) A} B \psi_{D}(\tau, s) d \tau .
$$

Comparing this last formula with (33), by uniqueness, one concludes that $K_{D} \phi_{D}(t, s)=\psi_{D}(t, s) K_{D}$.

\section{Conclusion}

The contribution of this paper was to inverse the Artstein transform and to derive an explicit expression of the corresponding stabilizing controller in terms of the history of the system state only. We illustrated how this new expression can be fruitful for theoretical analysis on linear systems with a constant delay in the input.

In a forthcoming work, we extend this inversion formula for infinite-dimensional linear systems, such as the heat equation with boundary delayed control.

\section{Appendix: Remarks on the numerical implementation}

For the sake of comparison, let us consider the control law (2)-(5).

It is interesting to notice that a differential implementation of this control law using (3) exhibits a very different behavior from the one based directly on the integral expression (2). Indeed, defining the variable $y=x(t+D)-$ $z(t)$, one concludes from (1) and (3) that $y$ satisfies the Ordinary Differential Equation $\dot{y}(t)=A y(t)$. Consequently, if the matrix $A$ is unstable and if $y(0) \neq 0$ (due to discretization issues in implementation of $z(0)$ for example) $y$ diverges numerically and, consequently, $x$ diverges as well.

${ }^{2}$ Recall that $\operatorname{rank}(A B) \leqslant \min (\operatorname{rank}(A), \operatorname{rank}(B))$ for two matrices $A$ and $B$. 
Conversely, if one wishes to implement directly (2),(5) under the integral form, there will still exist a robustness margin preserving numerical instabilities to occur, as established in the seminal paper [12]. Interestingly, in some sense, these differences are the twins of the ones existing between the original Smith Predictor and the Modified Smith Predictor, which is an equivalent representation of the Finite Spectrum Assignment as underlined in [22].

On the other hand, whatever the implementation method of (18) we follow (that is, using the fixed point equations (11)-(14) or their differential expression (19)-(21)) and the open-loop behavior of the plant (1), the stabilization properties of Theorem 2 only hold for a finite time $T^{*}$ in practice. Indeed, consider the effect of a numerical error (either resulting from an approximation of the fixed point equations or from an Euler approximation of the differential equations) as

$$
\hat{z}(t)=x(t)+\int_{0}^{t}\left[\Phi_{D}(t, s)+\epsilon(s)\right] x(s) d s=z(t)+\int_{0}^{t} \epsilon(s) x(s) d s
$$

which thus implies, with the control law $u(t)=K_{D} \hat{z}(t)$,

$$
\dot{x}(t)=\left(A+e^{-A D} B K_{D}\right) x(t)+B K_{D} \int_{0}^{t} \epsilon(t, s) x(s) d s
$$

Then, except if one is able to reduce the numerical errors in a way that $\epsilon$ tend to zero in a time-increasing compact set (i.e., make the numerical errors vanish with time, which is quite an unrealistic assumption), asymptotic convergence is not feasible.

This is the reason why we proposed in the simulation section to virtually reset the inital time $t_{0}$ periodically to reset the controller as well.

\section{References}

[1] Z. Artstein. Linear systems with delayed controls: a reduction. IEEE Transactions on Automatic Control, 27(4):869-879, 1982.

[2] N. Bekiaris-Liberis and M. Krstic. Nonlinear control under nonconstant delays, volume 25. SIAM, 2013.

[3] N. Bekiaris-Liberis and M. Krstic. Predictor-feedback stabilization of multiinput nonlinear systems. In 2015 54th IEEE Conference on Decision and Control (CDC), pages 7078-7083. IEEE, 2015.

[4] D. Bresch-Pietri and M. Krstic. Delay-adaptive control for nonlinear systems. IEEE Transactions on Automatic Control, 59(5):1203-1218, 2014.

[5] E. Fridman. Introduction to Time-Delay Systems: Analysis and Control. Brikhäuser, 2014. 
[6] K. Gu and S.-I. Niculescu. Survey on recent results in the stability and control of time-delay systems. Journal of Dynamic Systems, Measurement, and Control, 125(2):158-165, 2003.

[7] M. Henson and D. Seborg. Time delay compensation for nonlinear processes. Industrial \& engineering chemistry research, 33(6):1493-1500, 1994.

[8] C. Kravaris and R. Wright. Deadtime compensation for nonlinear processes. AIChE Journal, 35(9):1535-1542, 1989.

[9] M. Krstic. Lyapunov tools for predictor feedbacks for delay systems: Inverse optimality and robustness to delay mismatch. Automatica, 44(11):29302935, 2008.

[10] M. Krstic. Delay compensation for nonlinear, adaptive, and PDE systems. Springer, 2009.

[11] W. Kwon and A. Pearson. Feedback stabilization of linear systems with delayed control. IEEE Transactions on Automatic Control, 25(2):266-269, 1980.

[12] A. Manitius and A.W. Olbrot. Finite spectrum assignment problem for systems with delays. IEEE Transactions on Automatic Control, 24(4):541$552,1979$.

[13] F. Mazenc and M. Malisoff. Stabilization of nonlinear time-varying systems through a new prediction based approach. IEEE Transactions on Automatic Control, 2016.

[14] S. Mondié, M. Dambrine, and O. Santos. Approximation of control laws with distributed delays: a necessary condition for stability. Kybernetika, 38(5):541-551, 2002.

[15] S. Mondié and W. Michiels. Finite spectrum assignment of unstable timedelay systems with a safe implementation. IEEE Transactions on Automatic Control, 48(12):2207-2212, 2003.

[16] M. Nihtila. Finite pole assignment for systems with time-varying input delays. In Decision and Control, 1991., Proceedings of the 30th IEEE Conference on, pages 927-928. IEEE, 1991.

[17] A. Polyanin and A. V. Manzhirov. Handbook of Integral Equations, volume 67. CRC Press, 2007.

[18] A. Quadrat. A constructive algebraic analysis approach to artstein's reduction of linear time-delay systems. IFAC-PapersOnLine, 48(12):209-214, 2015 .

[19] J.-P. Richard. Time-delay systems: an overview of some recent advances and open problems. Automatica, 39(10):1667-1694, 2003. 
[20] J.-P. Richard, A. Goubet-Bartholomeüs, P.A. Tchangani, and M. Dambrine. Nonlinear delay systems: Tools for a quantitative approach to stabilization. In Stability and control of time-delay systems, pages 218-240. Springer, 1998.

[21] V Van Assche, M Dambrine, J-F Lafay, and J-P Richard. Some problems arising in the implementation of distributed-delay control laws. In Decision and Control, 1999. Proceedings of the 38th IEEE Conference on, volume 5, pages 4668-4672. IEEE, 1999.

[22] Qing-Chang Zhong. Robust control of time-delay systems. Springer Science \& Business Media, 2006.

[23] B. Zhou. Truncated predictor feedback for time-delay systems. Springer, 2014. 